Research Article

\title{
Development of a Pain Measurement Device Using Electrical Stimulation and Pressure: A Pilot Study
}

\author{
Seung Ho Lee, ${ }^{1}$ Ok-Kyun Kim, ${ }^{1}$ Hyung-Hwan Baik, ${ }^{1}$ and Ji-Hye Kim ${ }^{2}{ }^{2}$ \\ ${ }^{1}$ Department of Biochemistry \& Molecular Biology, Kyung Hee University Graduate School, Seoul 130-701, Republic of Korea \\ ${ }^{2}$ Department of Convergent Creative Manufacturing Engineering, Dankook University, Yongin, Gyeonggi-do, Republic of Korea
}

Correspondence should be addressed to Ji-Hye Kim; hlove3010@naver.com

Received 6 November 2017; Revised 31 January 2018; Accepted 19 February 2018; Published 25 March 2018

Academic Editor: Armando Ricciardi

Copyright (C) 2018 Seung Ho Lee et al. This is an open access article distributed under the Creative Commons Attribution License, which permits unrestricted use, distribution, and reproduction in any medium, provided the original work is properly cited.

\begin{abstract}
Understanding and precise assessment of pain level are key factors in rehabilitation therapy. Pain is a complex and subjective experience that is affected by an individual's emotion and health conditions. Various methods have been developed for quantitative evaluation of pain level; however, these methods have several drawbacks. In this work, we developed a pain measurement device for quantitative pain assessment. The system consists of two parts, a component for electrical stimulation and a pressure dolorimeter, for application of two different stresses. Regarding electrical stimulation, the degree of pain is assessed by the applied current. Skin resistance was also analyzed by applying current to remove the effects caused by skin conditions. The electrical stimulation did not induce any histological changes or inflammation in the tissues. Using the pressure dolorimeter, the pain level was assessed according to the degree of inflammation. This system could be used for the quantitative assessment of pain induced by inflammation, wounds, and other factors. Since the described system is the first of its kind, there are many problems that remain to be solved. However, with continuous development, our system could provide more accurate pain assessment by removing skin condition effects and through cross-validation.
\end{abstract}

\section{Introduction}

Assessment of a patient's pain scale is an important issue during rehabilitation. However, pain measurement is difficult because it is a complex sensation associated with personal characteristics and experience [1-4]. Various methods have been developed to assess pain scale, including Visual Analog Scale (VAS), Numerical Rating Scale (NRS), Verbal Rating Scale (VRS), FACES Pain Rating Scale (FPRS), and McGill Pain Questionnaire (MPQ) [5-9]. Among of them, VAS and FPRS have been mainly used in clinical settings $[10,11]$. The VAS is designed as a straight line of fixed length, 10 centimeters. The ends are defined as the extreme limits of the parameter to be measured orientated from "no pain" to the "worst imaginable pain." Patients mark on the line corresponding to the intensity of pain they currently experience. The NRS instructs the patients to choose a number from 0 to 10 that best describes their current pain intensity. 0 means "no pain" and 10 means "worst possible pain." The VRS is a method of pain evaluation that is expressed in words instead of numbers. Patients choose a sentence such as no pain, moderate pain, severe pain, very severe pain, and worst possible pain to describe their feeling of pain. The FPRS is a six-point pain scale with six different faces that represent increasing pain levels. Patients are asked to select the expression that best characterizes pain intensity, from "no pain" to "severe pain." All of these methods are subjective and depend on personal experience and emotion. Therefore, there are considerable individual variations in assessed pain that result in difficult quantitative evaluation.

Recently, a quantitative pain-assessing method using electrical stimulation was introduced [12-15]. PainVision ${ }^{\mathrm{TM}}$ devices measure perception threshold and pain produced by an electrical current. This system quantifies pain intensity by comparing the experienced pain with the intensity of electrical perceptions. The perception threshold indicates the minimal electric current sensed by the individual, and the pain produced is defined as the maximal electric 
current sensed by the individual. However, the skin resistance of an individual may affect the electrical measurement result [16-20].

The most important factors in the development of a pain measurement device are objectivity, accuracy, and quantitative assessment. PainVision is a system that allows patients to make measurements regardless of skin resistance. Skin resistance varies with the condition and environment of the patient. It is difficult to explain the correlation with previous measurements if the pain is measured neglecting skin resistance. But, our system measures from the patient's skin resistance before measuring the pain.

After determining the patient's baseline skin resistance value, the pain is measured. That is, the skin resistance value is a standard. When the reference value is prepared, a description of the correlation of the pain values measured before and after the pain measurement becomes possible.

In this work, we developed a pain measurement device that has two analysis methods, electrical stimulation and applying pressure, for more accurate cross-validation assessment. During electrical stimulation, electrical stress is applied on a nonpain site and then the observed stress can be compared to pain. The electrically stressed tissues were examined histologically to identify whether the electrical stimulation caused tissue damage. During the pressure evaluation, pain was assessed by applying pressure on the pain site. Inflammation was induced on the rat's hind paw by carrageenan, and then the inflamed hind paw was stimulated by a hand-type pressure stimulator. The pressure site was then compared with the inflammation levels.

\section{Material and Methods}

2.1. Principle and Components of the Pain Measurement Device. The pain measurement device was developed for quantitative pain analysis. The device is comprised of two major parts including a component for electrical stimulation and a pressure dolorimeter, as shown in Figure 1. Stimulation could be separately applied to the individual either electrically or via pressure. By comparing the response to the electrical stimulation and pressure, we could obtain more accurate results. During electrical stimulation, the pain experienced by patients is substituted for electrical stimulation; namely, the applied current value is translated to an experienced or experiencing pain value. Also, by evaluating the electrical stimulation and skin resistance at the same time, we expect to objectively evaluate pain despite varying conditions such as changes in skin and body, weather, or environmental conditions. While using the pressure dolorimeter, the pressure is applied to the pain site directly and onset of pain is measured by the amount of pressure.

The pain measurement device utilizes five buttons including power, ES (electrical stimulation), PS (pressure stimulation), EB (emergency button), and SS (stop switch). ES and PS are utilized for applying electrical and pressure stimulation to the individual. EB is a power shutdown button available in case of an emergency, and SS is a button used at the end of the pain measurements. The electrical stimulation component was connected with two electrodes for measuring

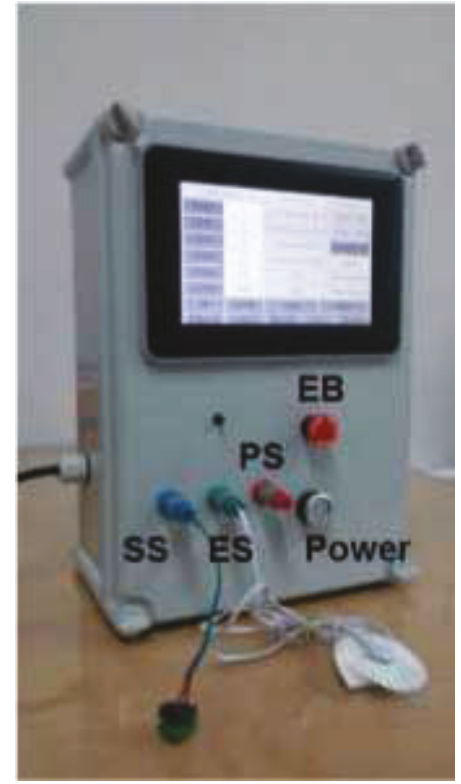

FIgURE 1: The pain measurement system with power button, electrical stimulation part (ES), pressure stimulation part (PS), stop switch (SS), and emergency button (EB).

the perceived current $(50 \mathrm{~Hz}$, pulse width $0.3 \mathrm{~ms})$ and pain threshold. The two electrodes are placed on a flat site, such as the flank of an animal or medial forearm of a human. The electrical stimulation system provides the same level of stimulation and stimulus intensity as pain; the Ad-fiber conducts the pain signal primarily (electrical signal). The pressure dolorimeter is connected to a tip-type pressure sensor of a hand stimulator. The stop switch was pushed when the participant felt pain.

2.2. Animal Model Preparation. Adult male Sprague-Dawley rats (Raon Bio. Inc., Yongin, Korea) weighing 250-350 g and 49-56 days old were kept under a $12 \mathrm{~h}$ light/12 h dark cycle (lights at $06: 00 \mathrm{~h}$ ) at $24 \pm 0.5^{\circ} \mathrm{C}$ in a central animal care facility. Water and rat chow were provided ad libitum until experiments began. All animal experiments were approved by the Committee of Animal Experiments in the College of Medicine, Kyung Hee University (KHUASP(SE)-15-084), and were treated in strict accordance with the National Institutes of Health Guide for the Care and Use of Laboratory Animals.

2.3. Histological Examination. Rats were either stimulated electrically or treated with carrageenan to induce pain. The inflammatory responses induced by the two different treatments were compared by histological evaluation. Rats were perfused intracardially with cold PBS containing heparin $(0.2 \mathrm{U} / \mathrm{ml})$ and $4 \%$ paraformaldehyde and then stocked in $4 \%$ paraformaldehyde. The stressed tissues resulting from electrical and carrageenan stimulation were bisected and placed in embedding cassettes. Each sample was embedded in paraffin wax and then sectioned into $5 \mu \mathrm{m}$ thick samples using a rotary microtome (HM340E, Microm, Walldorf, Germany). The sectioned sample was stained with hematoxylin and eosin (H\&E) and then analyzed under a Nikon 


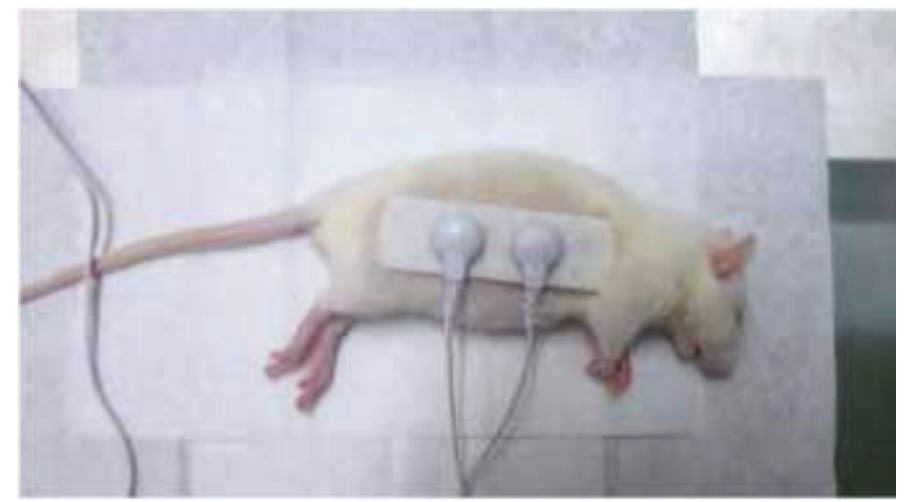

(a)

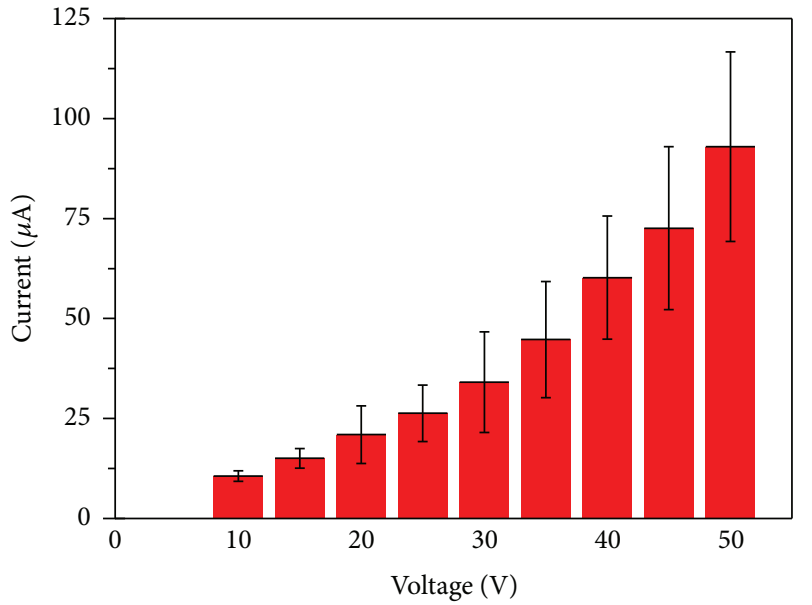

(b)

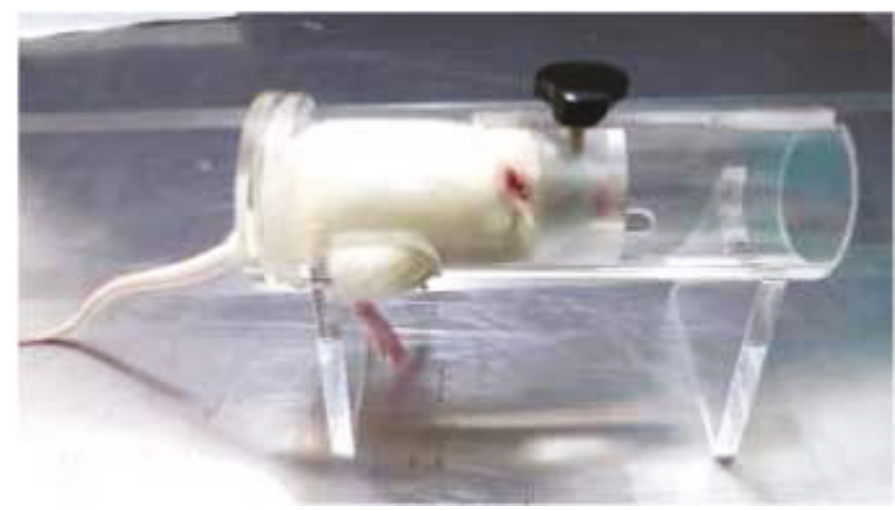

(c)

Figure 2: Method of pain evaluation measurements in animal model, using the pain measurement system. (a) The rat was attached to a bipolar electrode on the right flank side. (b) The current-voltage plot for measurement of skin resistance according to a gradually increasing pulsed voltage. (c) Rat was restrained for 1 2 $\mathrm{min}$ in a rat restrainer for pressure stimulation on carrageenan-induced paw edema.

inverted research microscope Ti-E and a Nikon Ds-Ril digital camera controlled by Nis-Elements (Br) software (Nikon Inc., Kawasaki, Japan).

2.4. Immunofluorescence Staining. The effects of electrical stimulation on inflammation and apoptosis of the tissues were investigated by immunofluorescence. The electrically stimulated flank tissue of rats was embedded in paraffin and sectioned into $4 \mu \mathrm{m}$ thick samples. For antigen retrieval, tissue sections were heated in TRS buffer for approximately ten min and then washed using distilled water three times. The samples were treated with $0.03 \% \mathrm{H}_{2} \mathrm{O}_{2}$ for inhibition of endogenous peroxidase activity and then washed with PBS solution. Then the samples were permeabilized with $0.5 \%$ Triton X-100 in PBS for ten min at room temperature and blocked with $5 \%$ goat and $1 \%$ bovine serum albumin in PBS for one hour. The samples were washed with PBS twice for ten min each. The samples were incubated overnight at $4^{\circ} \mathrm{C}$ with a primary antibody, either COX2 (diluted $1: 1000$ ) or caspase-3 (diluted 1:1000), diluted in 1\% bovine serum albumin in PBS. The samples were washed four times with PBS and then incubated in chronological order with secondary antibody (mouse and rabbit, Alexa Fluor 2nd ab, 1:500) diluted in 1\% BSA in PBS at room temperature in the dark for two hours. Finally, the samples were washed twice with PBS for two min and then covered with DAPI-containing mounting medium. Images were acquired using a Zeiss Axiovert microscope (Zeiss, Germany) and Axiovision Rel. 4.5 Analysis System (Zeiss, Germany).

\section{Results and Discussion}

3.1. Pain Measurement Scheme. For electrical stimulation, rats were anesthetized via intraperitoneal injection using chloral hydrate $(300 \mathrm{mg} / \mathrm{kg})$, and the hair of the rat was removed. The ECG electrode was attached to the right flank of the rat for electrical stimulation, as shown in Figure 2(a). The electrical stimulation was applied to the left flank of the rat. The applied voltage was gradually increased from 0 to $50 \mathrm{~V}$. The current value measured in each rat ranged from 12.5 to $92 \mu \mathrm{A}$ as shown in Figure 2(b). The responding behavior of the rat was not observed because the electrical stimulation was conducted under anesthesia. However, we expect that stimulation can always be measured regardless of the physical or skin condition by identifying the voltage, 


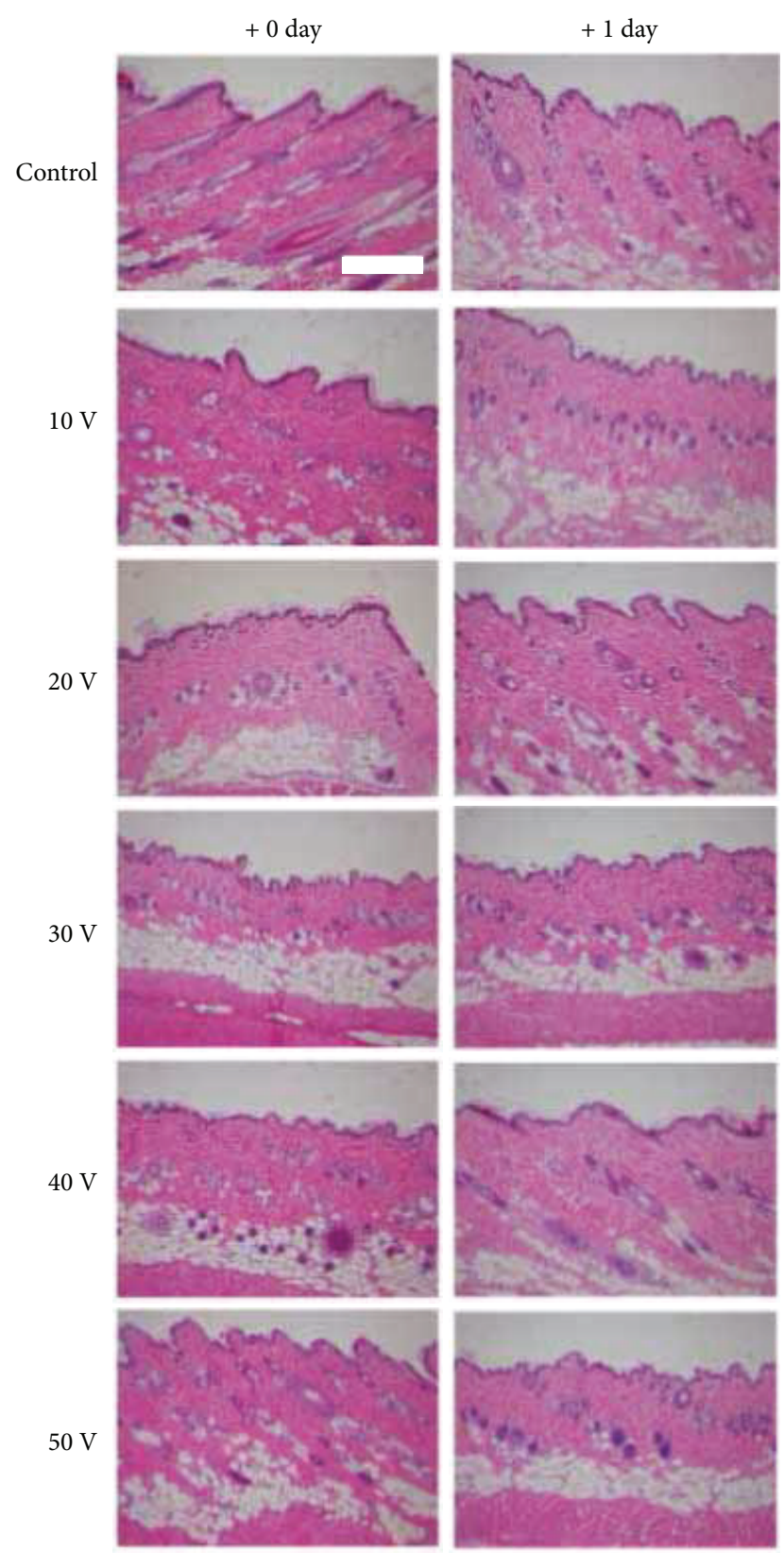

Figure 3: The histology of the right flank tissue sections from the control and $10 \mathrm{~V} \sim 50 \mathrm{~V}$ groups stained with hematoxylin and eosin. Scale bar: $100 \mu \mathrm{m}$.

current, and resistance related to the physiological factors of the rat.

In the pressure dolorimeter test, the rat was placed in a restraining chamber with no anesthetic, as shown in Figure 2(c). Inflammation was induced by carrageenan injection in the right hind paw. Carrageenan was used to confirm nociceptive pain. The equipment we built was also made to identify the nociceptive pain first. The inflammation levels were controlled by the carrageenan amount, which was increased from 100 to $200 \mu \mathrm{l}$ of a $1 \%(w / v)$ solution. $100 \mu \mathrm{l}$ of saline solution was injected on the intraplantar region of the left hind paw as a control. The inflamed right hind paw was stimulated by a hand-type pressure stimulator, as shown
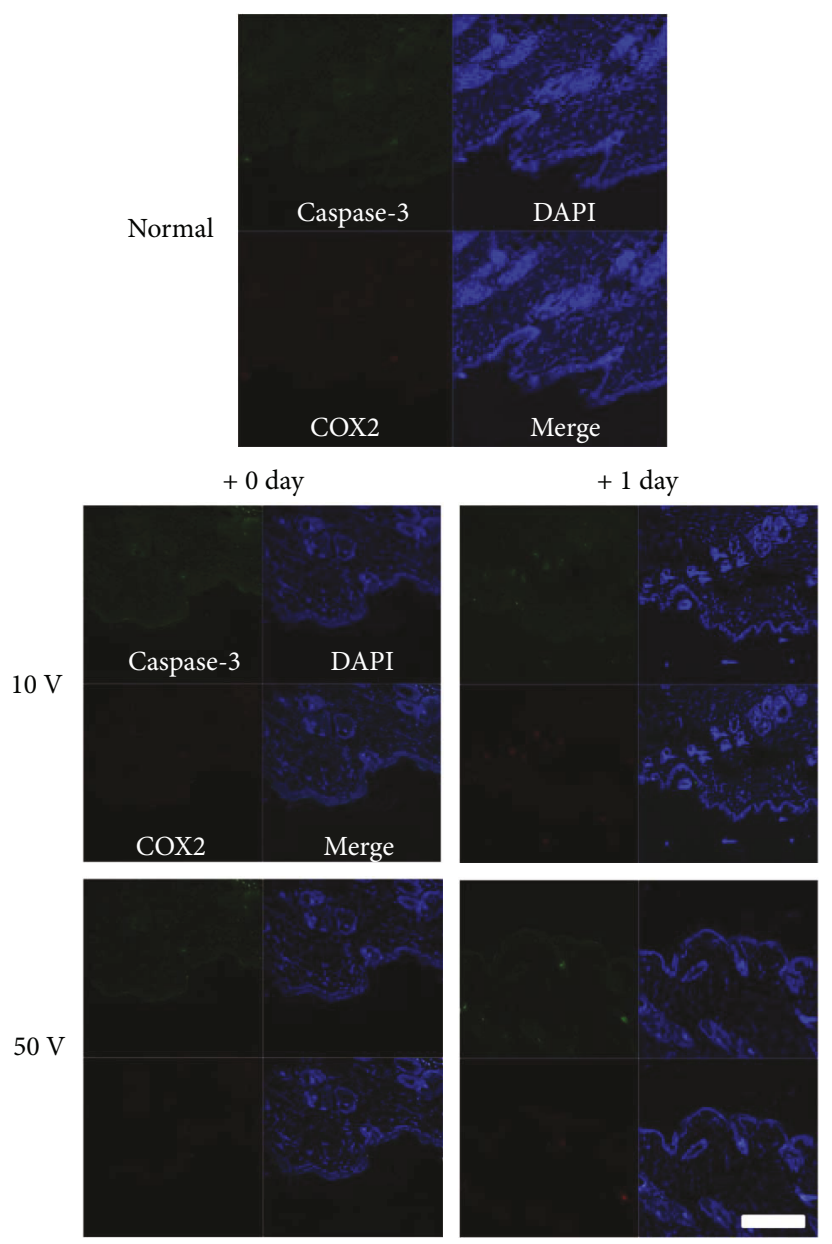

FIgURE 4: Representative immunofluorescence images of electricalstimulated rat tissues on each stained caspase-3, DAPI, and COX-2. Scale bar: $100 \mu \mathrm{m}$.

in Figure S1. The rat responded to the pressure stimulation. Our hypothesis is that the threshold of the pressure response in the rat depends on the degree of inflammation. Inflammation expression in the rat hind paw mimics the disease levels in clinical practice. Increases in hind paw volume and size according to inflammation were measured at each time point after the carrageenan injection. Hind paw volumes were measured by plethysmometry (model 7140 plethysmometer, Ugo Basile, Italy), and calipers (model CD-6P; Mitutoyo, Tokyo, Japan) were used to measure the width and thickness of the paws of each rat. The pressure stimulation was applied when the inflammation expression reached maximum. The rat response was observed by increasing the pressure level, and the pressure threshold was compared with the inflammation degree.

3.2. Electrical Stimulation. In order to verify that the electrical stimulation applied induced only pain without damage to the rat, the stimulated tissues were examined in terms of histology and inflammation. Figure 3 shows the macroscopic images of H\&E-stained tissues that were electrically stimulated by increasing voltage from 0 to $50 \mathrm{~V}$. One group of the tissues was stained immediately after the electrical 


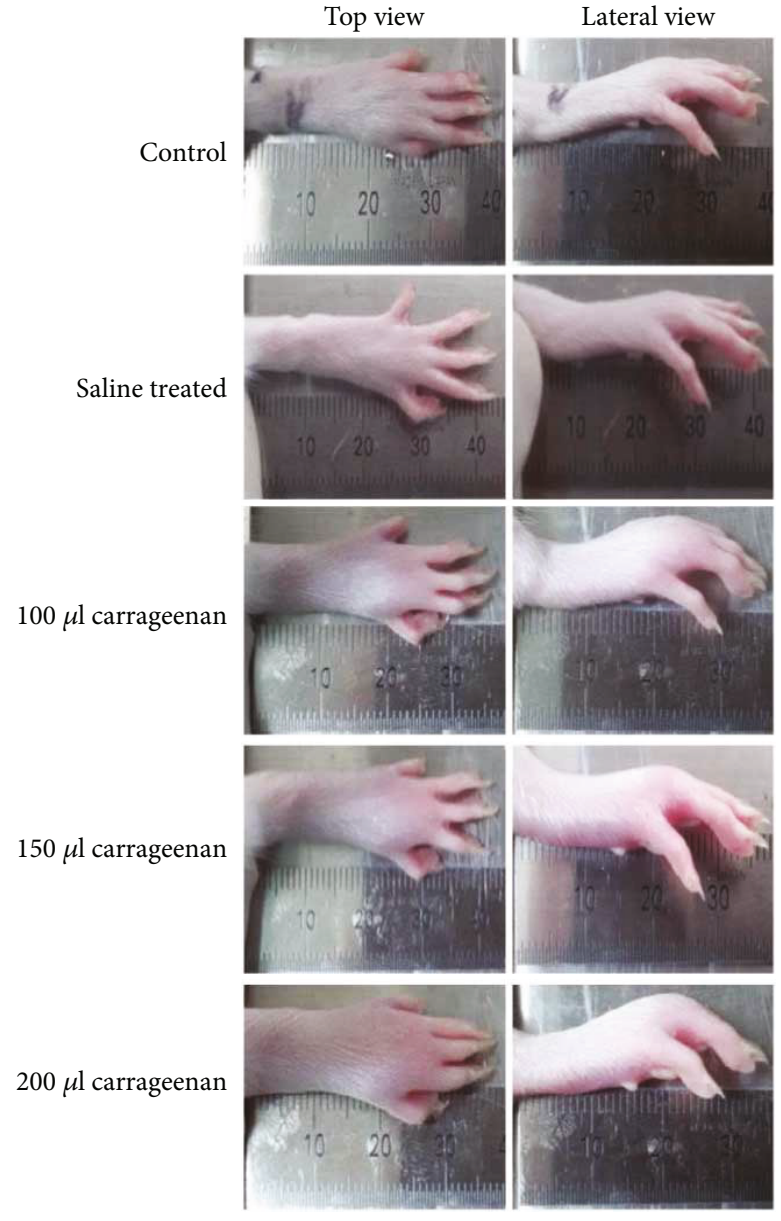

(a)

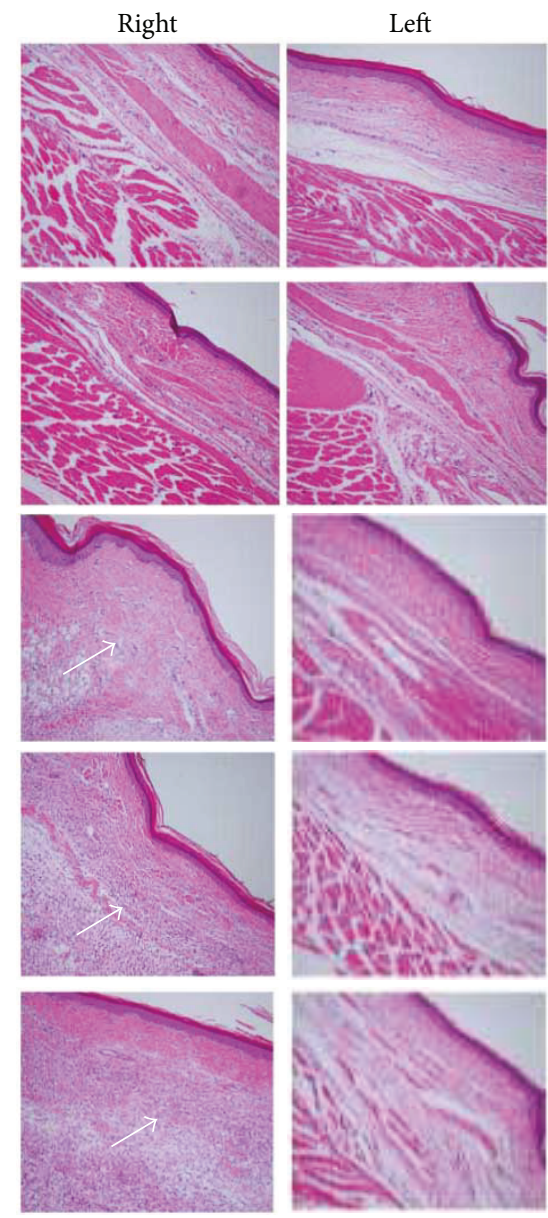

(b)

FiguRE 5: Effect of carrageenan-induced paw edema in rats. Typical representative (a) macroscopic photographs and (b) histology of hind paws from the control, saline-treated, $1 \% 100 \mu \mathrm{l}$ carrageenan-treated, $150 \mu \mathrm{l} 1 \%$ carrageenan-treated, and $1 \% 200 \mu \mathrm{l}$ carrageenantreated groups.

stimulation (left column), and the other was stained one day after stimulation (right column).

The control group showed normal flank tissue. No differences were observed in the electrically stressed tissues compared to the control tissue, even though the voltage increased to $50 \mathrm{~V}$. No abnormalities were observed 24 hours after electrical stimulation. Both inflammatory and apoptotic effects of the electrical stimulation were examined by double immunofluorescence staining for caspase-3 and COX-2. Figure 4 shows the results of double immunofluorescence staining for normal (nonstimulated) and stimulated (at 10 and $50 \mathrm{~V}$ ) tissues. There was no inflammation expression or apoptosis in the tissues immediately after electrical stimulation or after one day, confirming that electrical stimulation only caused pain and did not induce tissue damage.

3.3. Pressure Dolorimeter. The right hind paw of the rat was treated by carrageenan to induce inflammation, and the hind paw was photographed from top and lateral views, as shown in Figure 5. One group was not treated by carrageenan as a control, and the other was treated by saline as a negative control (Figure 5(a)). 1\% carrageenan was injected in varying amounts of $100 \mu \mathrm{l}, 150 \mu \mathrm{l}$, and $200 \mu \mathrm{l}$. The inflammatory symptoms of edema and redness were observed in all hind paws treated by carrageenan, while the control and salinetreated groups showed no symptoms. The inflammatory effect of carrageenan was clearly revealed by H\&E staining. As shown in Figure 5(b), the control and saline-treated groups showed a healthy tissue state. However, the accumulation of infiltrated inflammatory cells was observed in all carrageenan-treated groups as indicated by arrows. The right image is a right hind paw of a rat used for control, saline, and carrageenan, and the left image is a left hind paw of the rat in Figure 5(b).

During the inflammation progress, both the volume and size (width $\times$ height) of the hind paw were measured using plethysmometry and calipers. The measurement was conducted immediately after the saline and carrageenan injection and then continued for eight hours. In all carrageenan-treated groups, both the volume and size of hind paws progressively increased with time up to $6 \mathrm{~h}$; however, edema decreased with further increasing time (Figures 6(a) and 6(b)). The control and saline-treated groups showed no changes in volume and size. After measuring edema (Figures 6(a) and 6(b)) based 


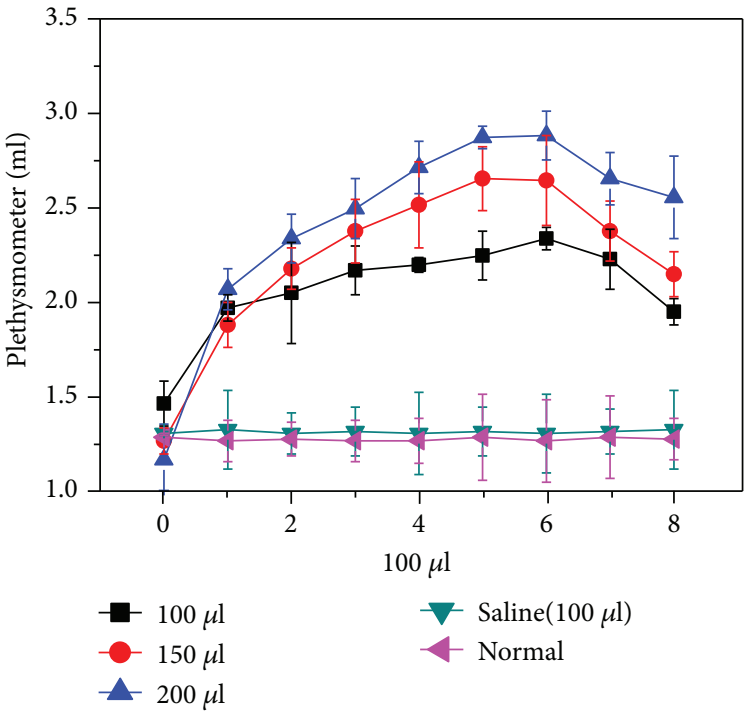

(a)

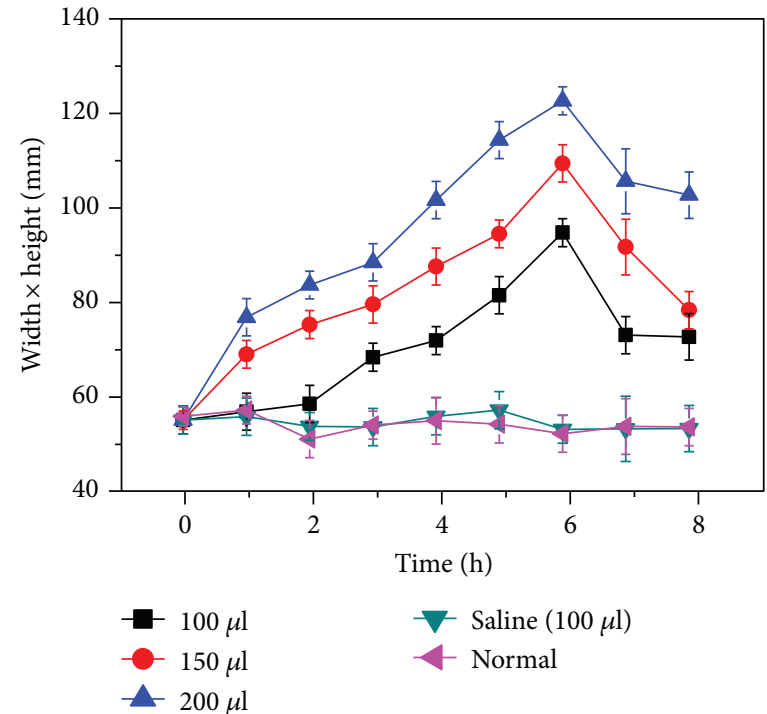

(b)

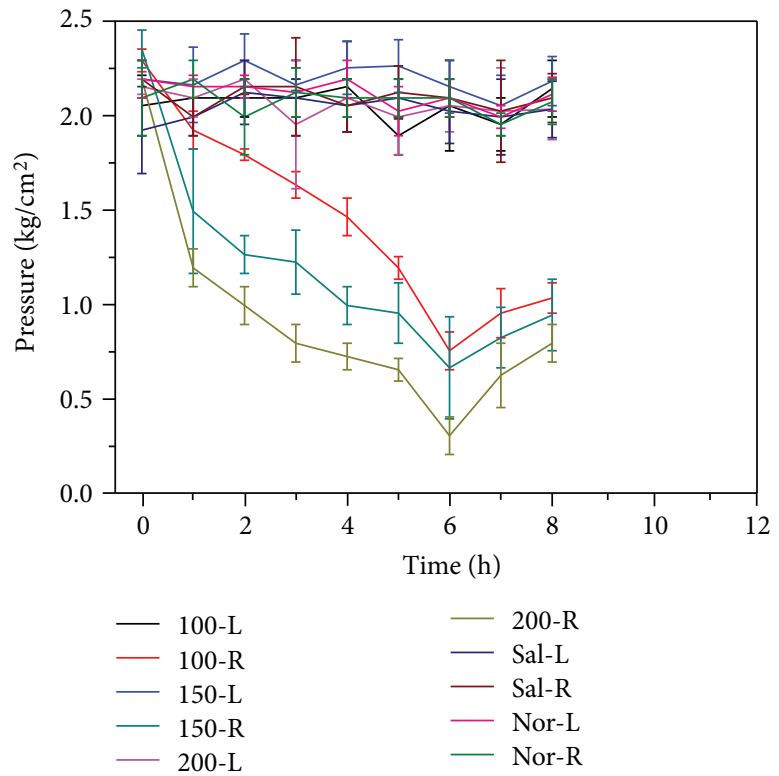

(c)

FIGURE 6: A time process showing the effects of carrageenan-induced paw volume using the (a) plethysmography, (b) calipers, and (c) pressure behavior according to carrageenan concentration.

on carrageenan injection concentration, we confirmed the rat's aspect of action (pressure) in the maximum state of edema $6 \mathrm{~h}$ after carrageenan injection (Figure 6(c)). This measurement was performed to collect a variety of information on the clinical inflammation state. In the control and saline groups, the pressure test results showed no significant changes. In the carrageenan-induced paw edema group, we were able to distinguish differences in pressure values depending on the observed inflammation. These results indicate that we could determine the degree of pain according to various inflammation levels. In this study, carrageenan was used to evaluate acute inflammation; assessment of chronic inflammation will be assessed using Complete Freund's Adjuvant.

\section{Conclusions}

We developed a pain measurement system for objective and quantitative pain assessment. For cross-validation, the system consisted of both electrical stimulation and pressure components. Before applying this system in a clinical setting, the safety and reliability of the system were confirmed using an animal model. The electrical stimulation did not cause any damage, including tissue inflammation and apoptosis. In the pressure test, the measured pressure values depended on the degree of inflammation. Our pain measurement system requires a short measurement time and did not induce inflammation when applied to rat tissue. At present, our research and development has been completed and ready 
for commercialization. The most commonly used part will be nociceptive pain. It is used in rehabilitation medicine department, and neurosurgical pain (neuropathic pain) will be applied in the future. Although many challenges remain, this pain measurement system may provide exciting opportunities for the diagnosis and treatment of various diseases.

\section{Conflicts of Interest}

The authors declare that they have no conflict of interest.

\section{Acknowledgments}

This research was supported by the "Software Convergence Technology Development Program," through the Ministry of Science, ICT and Future Planning (ITAS0177160110290001000200200) and the Ministry of Trade, Industry and Energy (MOTIE), Korea, through the Education Program for Creative and Industrial Convergence (Grant no. N0000717) and National Research Foundation of Korea (NRF) grant funded by the Korean government Ministry of Education, Science and Technology (no. 2011-0030072).

\section{Supplementary Materials}

Supplementary data associated with this article can be found in the online version. Figure S1: the hand-type pressure dolorimeter with pressure sensor and display components. Figure S2: an image showing the time course of carrageenan-induced paw edema after a $100 \mu \mathrm{l}$ injection volume. Figure S3: an image showing the time course of carrageenan-induced paw edema after a $150 \mu \mathrm{l}$ injection volume. Figure S4: an image showing the time course of carrageenan-induced paw edema after a $200 \mu \mathrm{l}$ injection volume. (Supplementary Materials)

\section{References}

[1] M. P. Jensen, P. Karoly, and S. Braver, "The measurement of clinical pain intensity: a comparison of six methods," Pain, vol. 27, no. 1, pp. 117-126, 1986.

[2] P. F. M. Verhaak, J. J. Kerssens, J. Dekker, M. J. Sorbi, and J. M. Bensing, "Prevalence of chronic benign pain disorder among adults: a review of the literature," Pain, vol. 77, no. 3, pp. 231-239, 1998.

[3] A. M. Elliott, B. H. Smith, K. I. Penny, W. Cairns Smith, and W. Alastair Chambers, "The epidemiology of chronic pain in the community," The Lancet, vol. 354, no. 9186, pp. 1248$1252,1999$.

[4] L. Litcher-Kelly, S. A. Martino, J. E. Broderick, and A. A. Stone, "A systematic review of measures used to assess chronic musculoskeletal pain in clinical and randomized controlled clinical trials," The Journal of Pain, vol. 8, no. 12, pp. 906-913, 2007.

[5] R. C. Wilson and P. W. Jones, "A comparison of the visual analogue scale and modified Borg scale for the measurement of dyspnoea during exercise," Clinical Science, vol. 76, no. 3, pp. 277-282, 1989.

[6] R. Melzack, "The McGill pain questionnaire: major properties and scoring methods," Pain, vol. 1, no. 3, pp. 277-299, 1975.
[7] A. K. Ismail, M. A. Abdul Ghafar, N. S. A. Shamsuddin, N. A. Roslan, H. Kaharuddin, and N. A. Nik Muhamad, "The assessment of acute pain in pre-hospital care using verbal numerical rating and visual analogue scales," The Journal of Emergency Medicine, vol. 49, no. 3, pp. 287-293, 2015.

[8] R. Melzack, "The short-form McGill pain questionnaire," Pain, vol. 30, no. 2, pp. 191-197, 1987.

[9] M. A. Ferreira-Valente, J. L. Pais-Ribeiro, and M. P. Jensen, "Validity of four pain intensity rating scales," Pain, vol. 152, no. 10, pp. 2399-2404, 2011.

[10] H. M. McCormack, D. J. de L. Horne, and S. Sheather, "Clinical applications of visual analogue scales: a critical review," Psychological Medicine, vol. 18, no. 4, pp. 1007-1019, 1988.

[11] C. L. Hicks, C. L. von Baeyer, P. A. Spafford, I. van Korlaar, and B. Goodenough, "The faces pain scale - revised: toward a common metric in pediatric pain measurement," Pain, vol. 93, no. 2, pp. 173-183, 2001.

[12] T. Fukada, H. Iwakiri, and M. Ozaki, "A randomised doubleblind crossover trial of the potential analgesic effect of a transdermal nicotine patch in non-smokers based on objective and subjective assessment," European Journal of Anaesthesiology, vol. 28, no. 8, pp. 592-596, 2011.

[13] A. Kanai, T. Horie, and H. Okamoto, "Change of the affected skin temperature in the sub-acute stage predicts the pain intensity and disability in patients with post-her petic neuralgia," in Proceedings of the American Society of Anesthesiologists (ASA) 2012 Annual Meeting, pp. 13-17, Washington, DC, USA, October 2012.

[14] N. Maekawa, M. Morimoto, M. Morimoto, T. Uchida, T. Umeda, and Y. Koga, "Can we evaluate pain with PainVision $^{\circledR}$, a device for quantitative analysis of perception and pain? A feasibility study of pain in herpes zoster patients," The Journal of Japan Society for Clinical Anesthesia, vol. 29, no. 7, pp. 824-828, 2009.

[15] H. Matsumura, R. Imai, M. Gondo, and K. Watanabe, "Evaluation of pain intensity measurement during the removal of

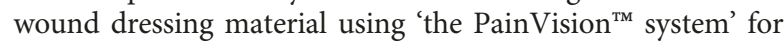
quantitative analysis of perception and pain sensation in healthy subjects," International Wound Journal, vol. 9, no. 4, pp. 451-455, 2012.

[16] P. A. Obrist, "Skin resistance levels and galvanic skin response: unilateral differences," Science, vol. 139, no. 3551, pp. 227-228, 1963.

[17] S. H. Shah, P. S. Nahar, and P. Balasubramanium, "Effect of gender differences on pain parameters and galvanic skin resistance in response to acute cold pain," Indian Journal of Basic \& Applied Medical Research, vol. 1, pp. 193-198, 2012.

[18] S. Kramer, K. Winterhalter, G. Schober et al., "Characteristics of electrical skin resistance at acupuncture points in healthy humans," Journal of Alternative and Complementary Medicine, vol. 15, no. 5, pp. 495-500, 2009.

[19] W. Li, "Modelling methods for in vitro biomechanical properties of the skin: a review," Biomedical Engineering Letters, vol. 5, no. 4, pp. 241-250, 2015.

[20] K. Kwak, H. G. Kim, and D. W. Kim, "Variation of ankle biomechanical property according to vibro-perception threshold and vibration frequency," Biomedical Engineering Letters, vol. 6, no. 1, pp. 16-25, 2016. 


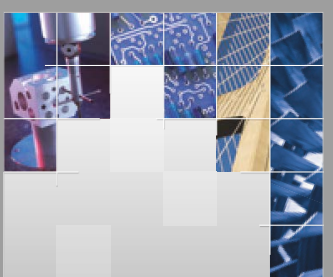

\section{Enfincering}
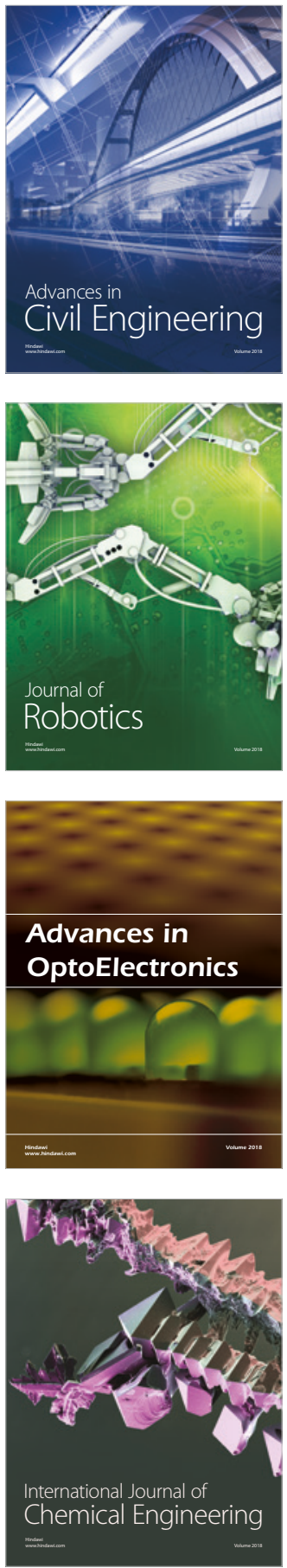

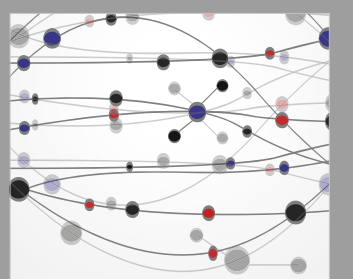

\section{Rotating \\ Machinery}

The Scientific World Journal

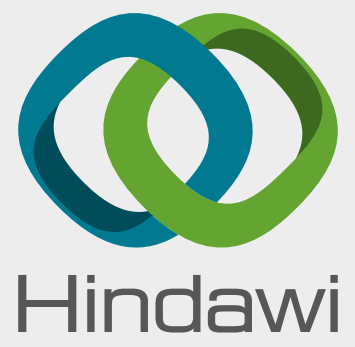

Submit your manuscripts at

www.hindawi.com
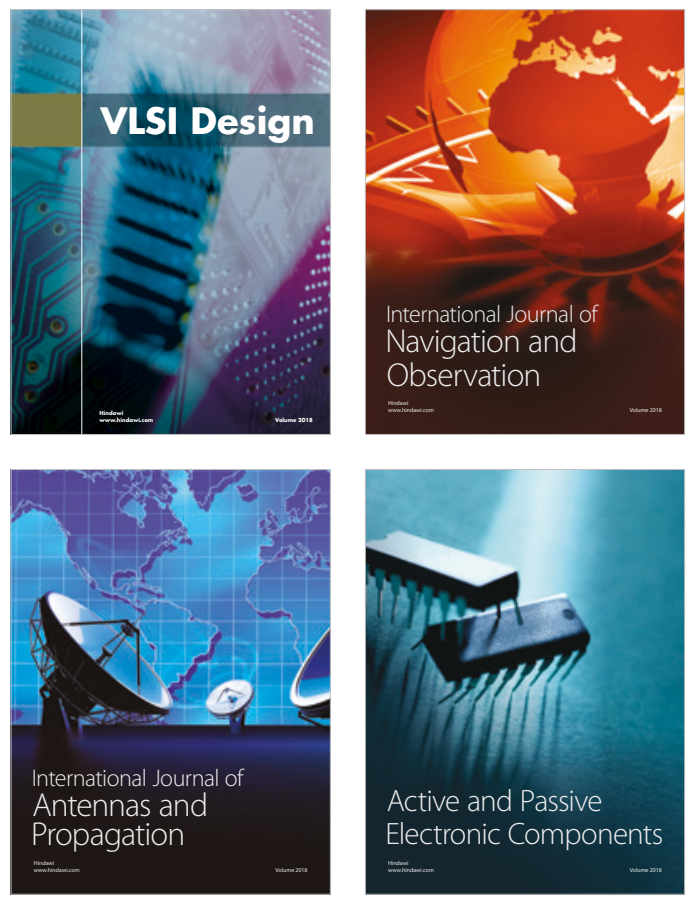
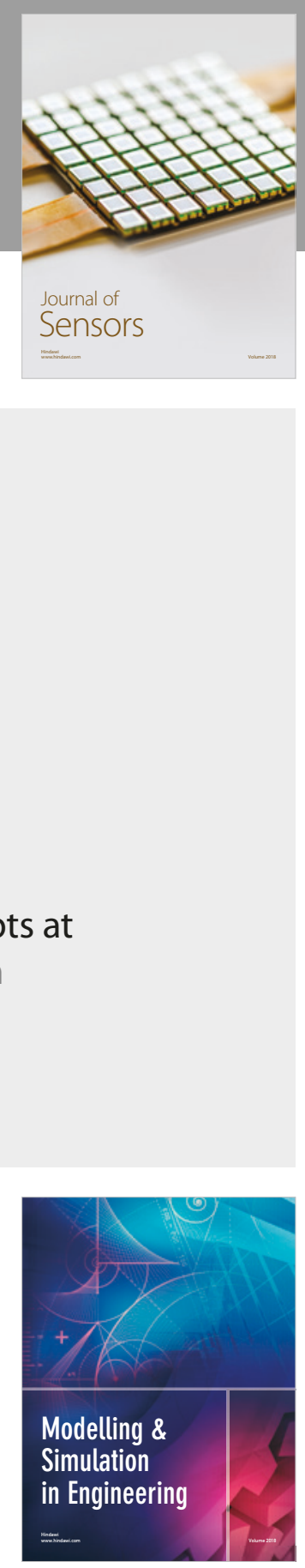

\section{Advances \\ Multimedia}
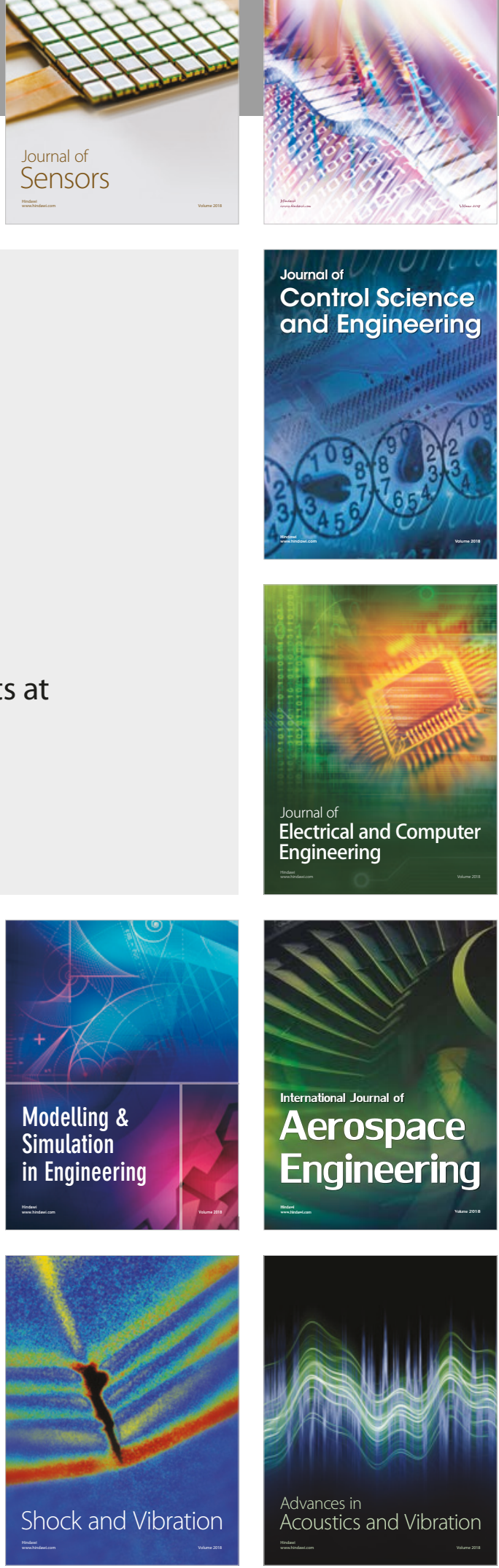